\title{
Association between Inhaled Corticosteroid Use and SARS-CoV-2 Infection: A Nationwide Population-Based Study in South Korea
}

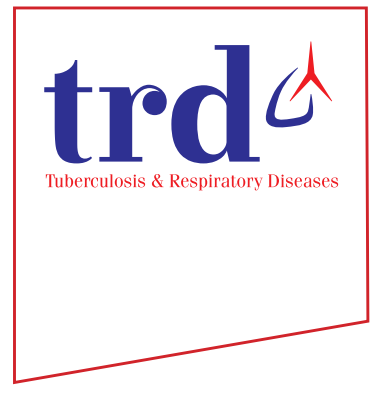

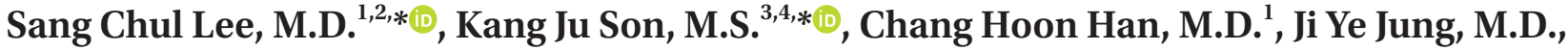 \\ Ph.D. ${ }^{-}$(i) and Seon Cheol Park, M.D., Ph.D. ${ }^{1}{ }^{(\mathbb{D}}$ \\ ${ }^{1}$ Division of Pulmonology, Department of Internal Medicine, National Health Insurance Service Ilsan Hospital, Goyang, \\ ${ }^{2}$ Graduate School, Yonsei University College of Medicine, Seoul, ${ }^{3}$ Department of Research and Analysis, National Health \\ Insurance Service Ilsan Hospital, Goyang, ${ }^{4}$ Department of Biostatistics and Computing, Yonsei University Graduate School, \\ Seoul, ${ }^{5}$ Division of Pulmonology, Department of Internal Medicine, Severance Hospital, Yonsei University College of Medicine, \\ Seoul, Republic of Korea
}

Background: Although it is known that inhaled corticosteroid (ICS) use may increase the risk of respiratory infection, its influence on the risk of severe acute respiratory syndrome coronavirus 2 (SARS-CoV-2) infection remains unknown. Thus, the aim of this study was to investigate the association between ICS use and the positivity of SARS-CoV-2 infection among patients with chronic respiratory diseases.

Methods: Nationwide data of 44,968 individuals with chronic respiratory diseases tested for SARS-CoV-2 until May 15, 2021 were obtained from the Ministry of Health and Welfare and Health Insurance Review and Assessment Service in Korea. The positivity of SARS-CoV-2 infection was retrospectively analysed according to the prescription, type, and dose of ICS taken one year before SARS-CoV-2 test.

Results: Among 44,968 individuals tested, 931 (2.1\%) were positive for SARS-CoV-2. A total of 7,019 patients (15.6\%) were prescribed ICS one year prior to being tested for SARS-CoV-2. Low, medium, and high doses of ICS were prescribed in $7.5 \%, 1.6 \%$, and $6.5 \%$ of total cases, respectively. Among types of ICS, budesonide, fluticasone, beclomethasone, and ciclesonide were prescribed in $3.7 \%, 8.9 \%, 2.3 \%$, and $0.6 \%$ of total cases, respectively. A multivariate analysis showed no significant increase in infection with ICS use (odds ratio, 0.84; 95\% confidence interval, 0.66-1.03). Moreover, there were no associations between the positivity of infection and the dose or type of ICS prescribed.

Conclusion: Prior ICS use did not increase the positivity for SARS-CoV-2 infection. Moreover, different doses or types of ICS did not affect this positivity.

Keywords: Inhaled Corticosteroid; Severe Acute Respiratory Syndrome Coronavirus 2; Chronic Respiratory Diseases; Risk

Address for correspondence: Ji Ye Jung, M.D., Ph.D.

Division of Pulmonology, Department of Internal Medicine, Severance Hospital, Yonsei University College of Medicine, 50-1 Yonsei-ro, Seodaemun-gu, Seoul 03722, Republic of Korea

Phone: 82-2-2228-1980, Fax: 82-2-393-6884, E-mail: STOPYES@yuhs.ac

Address for correspondence: Seon Cheol Park, M.D., Ph.D.

Division of Pulmonology, Department of Internal Medicine, National Health Insurance Service Ilsan Hospital, 100 Ilsan-ro, Ilsandong-gu, Goyang 10444 , Republic of Korea

Phone: 82-31-900-0263, Fax: 82-31-900-3663, E-mail: parksc@nhimc.or.kr

*Sang Chul Lee and Kang Ju Son contributed equally to this work.

Received: Jul. 1, 2021, Revised: Oct. 23, 2021, Accepted: Nov. 24, 2021, Published online: Nov. 30, 2021

(c) It is identical to the Creative Commons Attribution Non-Commercial License (http://creativecommons.org/licenses/by-nc/4.0/) 


\section{Introduction}

At the end of December 2019, an unexplained pneumonia emerged in Wuhan, Hubei Province, China. This disease was found to be caused by a novel coronavirus, severe acute respiratory syndrome coronavirus 2 (SARS-CoV-2) ${ }^{1}$. As of December 11, 2021, SARS-CoV-2 has resulted in over 267 million infections and over 5 million deaths worldwide ${ }^{2}$. Patients with mild symptoms such as fever and cough often recover from the SARS-CoV-2 infection. However, some die from severe complications $^{3}$. In patients with SARS-CoV-2, the presence of respiratory disease is associated with poor prognosis. Based on chest computed tomography, $86.2 \%$ of hospitalized patients show abnormalities ${ }^{4,5}$.

Inhaled corticosteroids (ICS) are essential drugs for major respiratory diseases such as asthma and chronic obstructive pulmonary disease (COPD). However, ICS may increase the risk of respiratory infection. A meta-analysis has reported that the use of ICS alone or in combination may increase the risk of pneumonia in COPD patients ${ }^{6}$. Regarding asthma, some studies have reported that ICS use may increase the risk of pneumonia, although this is controversial ${ }^{7,8}$. ICS may also increase the risk of tuberculosis or nontuberculous mycobacterial infection ${ }^{9,10}$.

ICS are frequently prescribed to patients with respiratory diseases who may be at a high risk for severe SARS-CoV-2 infection. However, the influence of ICS use on the risk of SARS$\mathrm{CoV}-2$ infection remains unknown. Thus, the purpose of this study was to evaluate the association between ICS use and the positivity of SARS-CoV-2 infection in patients with chronic respiratory diseases.

\section{Materials and Methods}

\section{Data source}

For a global research collaboration on SARS-CoV-2, the Ministry of Health and Welfare and Health Insurance Review and Assessment Service (HIRA) of Korea shared nationwide data of SARS-CoV-2 with researchers. The HIRA dataset was based on the insurance benefit claim sent to the HIRA. It comprised all cases tested for SARS-CoV-2 in Korea. This dataset includes various health-related information, including sociodemographic characteristics, health care utilization including diagnosis and medications, and survival status.

\section{Case definition}

Among a total of 234,427 individuals tested for SARS-CoV-2, 44,968 subjects who had comorbidity of chronic respiratory diseases (asthma, J45-46; COPD, J43-44; bronchiectasis J47; according to the International Classification of Diseases, 10th revision) in the first year before the test of SARS-CoV-2 were included in this study using HIRA dataset until May 15, 2020. SARS-CoV-2 infection was confirmed by a reverse transcription polymerase chain reaction test for SARS-CoV-2 using nasopharyngeal swab or sputum specimens. Those with negative results were considered as negative controls for SARSCoV-2 infection.

Exacerbations in previous 1 year before the test of SARS$\mathrm{CoV}-2$ were classified as moderate, emergency room visit, and hospitalization. Moderate exacerbation was defined as presence of chronic respiratory diseases diagnostic code plus the use of systemic corticosteroid or antibiotics.

Drug adherence was assessed with medication possession ratio (MPR) for asthma medications ${ }^{11,12}$. MPR was calculated as the sum of the day's supply for medication fills divided by the time from first fill in previous 1 year until the test of SARSCoV-2. MPR ratio was categorized into three adherence groups as follows: low adherence (MPR $<50 \%$ ), medium adherence (MPR 50\%-79\%), and high adherence (MPR $\geq 80 \%$ ).

\section{Demographic factors}

Information on sex, age, region of residence, and medical aid were taken at the time of SARS-CoV-2 diagnosis. Data regarding comorbidity and ICS prescription were collected during the first year before the test of SARS-CoV-2. The Charlson Comorbidity Index (CCI) for stratifying the risk of mortality or the resource use of patients based on comorbidities was recorded as previously described ${ }^{13}$. Comorbidities were categorized into heart disease (ischemic heart disease, I2025; congestive heart failure, I50), diabetes mellitus (E10-14), hypertension (I10), and cancer (C00-97) according to the International Classification of Diseases, 10th revision.

\section{Outcomes}

Among all individuals tested for SARS-CoV-2, the case group included patients with SARS-CoV-2 infection and the control group included patients without SARS-CoV-2 infection. The positivity of SARS-CoV-2 infection was analysed according to the prescription, type, and dose of ICS taken 1 year before the SARS-CoV-2 test. The type of ICS was categorized into budesonide, fluticasone, beclomethasone, and ciclesonide. The dose of ICS was calculated as the average daily consumption. It was categorized into low, medium, and high doses according to the Global Initiative for Asthma (GINA) report $^{14}$.

\section{Statistical analysis}

Descriptive statistics were performed for all variables. Differences between the two groups (case and control) were assessed by chi-squared test for categorical variables. Mul- 
Table 1. Baseline demographics of individuals tested for SARS-CoV-2

\begin{tabular}{|c|c|c|c|c|}
\hline Variable & $\begin{array}{c}\text { Total } \\
(n=44,968)\end{array}$ & $\begin{array}{l}\text { Positive for SARS-CoV-2 } \\
\qquad(n=931)\end{array}$ & $\begin{array}{l}\text { Negative for SARS-CoV-2 } \\
\qquad(n=44,037)\end{array}$ & p-value \\
\hline Male sex & $22,210(49.4)$ & $355(38.1)$ & 21,855 (49.6) & $<0.001$ \\
\hline Age, yr & & & & $<0.001$ \\
\hline $0-19$ & $5,363(11.9)$ & $62(6.7)$ & $5,301(12.0)$ & \\
\hline $20-39$ & $8,753(19.5)$ & $205(22.0)$ & $8,548(19.4)$ & \\
\hline $40-59$ & $8,901(19.8)$ & 269 (28.9) & $8,632(19.6)$ & \\
\hline $60-79$ & $14,546(32.3)$ & $298(32.0)$ & $14,248(32.4)$ & \\
\hline$\geq 80$ & 7,405 (16.5) & $97(10.4)$ & $7,308(16.6)$ & \\
\hline Region of residence & & & & $<0.001$ \\
\hline \multicolumn{5}{|l|}{ North-western } \\
\hline Seoul $^{*}$ & $13,114(29.2)$ & $72(7.7)$ & $13,042(29.6)$ & \\
\hline Incheon* & $2,341(5.2)$ & $18(1.9)$ & $2,323(5.3)$ & \\
\hline Daejeon* & $1,557(3.5)$ & $11(1.2)$ & $1,546(3.5)$ & \\
\hline Gyeonggi $^{\dagger}$ & $9,463(21.0)$ & $72(7.7)$ & $9,391(21.3)$ & \\
\hline Chungcheongbuk $^{\dagger}$ & $1,175(2.6)$ & $21(2.3)$ & $1,154(2.6)$ & \\
\hline Chungcheongnam $^{\dagger}$ & $2,523(5.6)$ & $46(4.9)$ & $2,477(5.6)$ & \\
\hline \multicolumn{5}{|l|}{ North-eastern } \\
\hline Gangwon $^{\dagger}$ & $1,127(2.5)$ & $21(2.3)$ & $1,106(2.5)$ & \\
\hline \multicolumn{5}{|l|}{ South-eastern } \\
\hline Daegu* & $4,370(9.7)$ & $422(45.3)$ & $3,948(8.9)$ & \\
\hline Busan* & $2,515(5.6)$ & $15(1.6)$ & $2,500(5.7)$ & \\
\hline Ulsan* & $742(1.7)$ & $4(0.4)$ & $738(1.7)$ & \\
\hline Gyeongsangbuk $^{\dagger}$ & $1,626(3.6)$ & $153(16.4)$ & $1,473(3.3)$ & \\
\hline Gyeongsangnam $^{\dagger}$ & $1,585(3.5)$ & $36(3.9)$ & $1,549(3.5)$ & \\
\hline \multicolumn{5}{|l|}{ South-western } \\
\hline Gwangju* & $712(1.6)$ & $11(1.2)$ & $701(1.6)$ & \\
\hline Jeollabuk $^{\dagger}$ & $1,082(2.4)$ & $23(2.5)$ & $1,059(2.4)$ & \\
\hline Jeollanam $^{\dagger}$ & $712(1.6)$ & $6(0.6)$ & $706(1.6)$ & \\
\hline Jeju Island $^{\dagger}$ & $324(0.7)$ & $0(0)$ & $324(0.7)$ & \\
\hline Medical-aid beneficiaries & $3,897(8.7)$ & $82(8.8)$ & $3,815(8.7)$ & 0.877 \\
\hline \multicolumn{5}{|l|}{ Comorbidities } \\
\hline Heart disease & $9,508(21.1)$ & $121(13.0)$ & $9,387(21.3)$ & $<0.001$ \\
\hline Diabetes mellitus & $13,371(29.7)$ & $210(22.6)$ & $13,161(29.9)$ & $<0.001$ \\
\hline Hypertension & $18,182(40.4)$ & $294(31.6)$ & $17,888(40.6)$ & $<0.001$ \\
\hline Cancer & $7,092(15.8)$ & $63(6.8)$ & $7,029(15.9)$ & $<0.001$ \\
\hline Charlson comorbidity index & & & & $<0.001$ \\
\hline 1 & $15,084(33.5)$ & $374(40.2)$ & $14,710(33.4)$ & \\
\hline 2 & $7,636(16.9)$ & $182(19.6)$ & $7,454(16.9)$ & \\
\hline$\geq 3$ & $22,248(49.5)$ & $375(40.3)$ & $21,873(49.7)$ & \\
\hline
\end{tabular}


Table 1. Continued

\begin{tabular}{|c|c|c|c|c|}
\hline Variable & $\begin{array}{c}\text { Total } \\
(n=44,968)\end{array}$ & $\begin{array}{l}\text { Positive for SARS-CoV-2 } \\
\qquad(\mathrm{n}=931)\end{array}$ & $\begin{array}{l}\text { Negative for SARS-CoV-2 } \\
(n=44,037)\end{array}$ & p-value \\
\hline \multicolumn{5}{|c|}{ Exacerbations in previous 1 year } \\
\hline Moderate exacerbations & & $0.42(0.82)$ & $0.51(0.95)$ & $<0.001$ \\
\hline 0 & $30,695(68.3)$ & $667(71.6)$ & $30,028(68.2)$ & 0.025 \\
\hline$\geq 1$ & $14,273(31.7)$ & $264(28.4)$ & $14,009(31.8)$ & 0.025 \\
\hline Emergency room visit & & $0.01(0.1)$ & $0.06(0.41)$ & $<0.001$ \\
\hline 0 & $42,935(95.5)$ & $922(99)$ & $42,013(95.4)$ & $<0.001$ \\
\hline$\geq 1$ & $2,033(4.5)$ & $9(1)$ & $2,024(4.6)$ & $<0.001$ \\
\hline Hospitalization & & $0.06(0.4)$ & $0.13(0.53)$ & $<0.001$ \\
\hline 0 & $41,166(91.5)$ & $896(96.2)$ & $40,270(91.5)$ & $<0.001$ \\
\hline$\geq 1$ & $3,802(8.5)$ & $35(3.8)$ & $3,767(8.6)$ & $<0.001$ \\
\hline
\end{tabular}

Values are presented as number (\%).

*Cities in South Korea. ${ }^{\dagger}$ Provinces in South Korea.

SARS-CoV-2: severe acute respiratory syndrome coronavirus 2.

tiple logistic regression analysis was performed to determine whether ICS was associated with the positivity of SARS-CoV-2 infection. Three models were used to analyze the positivity of infection according to ICS prescription, type, and dose. The positivity of infection is presented as odds ratio (OR) with 95\% confidence interval (CI). A p-value $<0.05$ was considered statistically significant. All statistical analyses were performed with SAS version 9.4 (SAS Institute Inc., Cary, NC, USA).

\section{Ethics statement}

This study was approved by the Institutional Review Board of the National Health Insurance Service of Ilsan Hospital. It adhered to the tenets of the Declaration of Helsinki (NHIMC 2020-04-008). Written informed consent was waived as data used were de-identified in the database.

\section{Results}

\section{Baseline demographics of individuals tested for SARS-CoV-2}

A total of 44,968 individuals were included. Of them, 931 (2.1\%) were positive for SARS-CoV-2 (Table 1). A total of 355 $(0.7 \%)$ males and 576 (1.3\%) females were positive for SARSCoV-2. In terms of age group, individuals aged 60-79 years had a higher proportion (32.3\%) of those tested for SARSCoV-2, followed by individuals aged $40-59$ years (19.8\%) and 20-39 years (19.5\%). When 16 regions (cities and provinces) in South Korea were compared, the South-Eastern region had high rates of SARS-CoV-2 infection. In Seoul, the number of SARS-CoV-2 cases was relatively low considering the large number of individuals tested. Among the four types of comorbidities, patients with any comorbidity tended to have a lower probability of SARS-CoV-2 infection in univariate analysis. This trend was similar in the analysis according to CCI. The higher the CCI, the lower the probability of SARS-CoV-2 infection. All types of exacerbations in previous 1 year before SARS-CoV-2 test were more frequency observed in those with a negative result for SARS-CoV-2 than those with a positive result for SARS-CoV-2.

\section{Patterns of ICS use in the past 1 year}

A total of 7,019 patients (15.6\%) were prescribed ICS 1 year prior to being tested for SARS-CoV-2 (Table 2). Low, medium, and high doses of ICS were prescribed for $7.5 \%, 1.6 \%$, and $6.5 \%$ of total cases, respectively. In both groups, low MPR was dominant. Most patients were prescribed with one type of ICS. Budesonide, fluticasone, beclomethasone, and ciclesonide were prescribed for $3.7 \%, 8.9 \%, 2.3 \%$, and $0.6 \%$ of total cases, respectively.

\section{Multivariate analysis for ICS use and positivity of SARS-CoV-2 infection}

A multivariate analysis was performed to assess whether ICS use was associated with SARS-CoV-2 infection (Table 3 , Supplementary Table S1). In model 1, individuals who were taking ICS were compared with those who were not taking ICS. The positivity of developing SARS-CoV-2 infection was not associated with the use of ICS (OR, 0.84; 95\% CI, 0.68-1.03). There was no association between the positivity of SARS-CoV-2 infection and ICS dose in model 2. When positivity of infection was analyzed according to ICS type, no type of 
Table 2. Patterns of inhaled corticosteroid use in the past 1 year

\begin{tabular}{|c|c|c|c|c|}
\hline Variable & $\begin{array}{c}\text { Total } \\
(n=44,968)\end{array}$ & $\begin{array}{l}\text { Positive for SARS-CoV-2 } \\
\qquad(n=931)\end{array}$ & $\begin{array}{l}\text { Negative for SARS-CoV-2 } \\
\qquad(n=44,037)\end{array}$ & p-value \\
\hline Inhaled corticosteroid used & $7,019(15.6)$ & $114(12.2)$ & $6,905(15.7)$ & 0.004 \\
\hline Dose & & & & $<0.001$ \\
\hline Low & $3,349(47.7)$ & $54(47.3)$ & $3,295(47.7)$ & \\
\hline Medium & $728(10.4)$ & $10(8.8)$ & $718(10.4)$ & \\
\hline High & $2,942(41.9)$ & $50(43.9)$ & $2,892(41.9)$ & \\
\hline ICS MPR* & & & & 0.723 \\
\hline Low & $6,980(99.4)$ & $114(100)$ & $6,866(99.4)$ & \\
\hline Medium & $14(0.2)$ & $0(0)$ & $14(0.2)$ & \\
\hline High & $25(0.4)$ & $0(0)$ & $25(0.4)$ & \\
\hline No. of ICS types & & & & 0.491 \\
\hline 1 & $6,399(91.2)$ & $106(93)$ & $6,293(91.1)$ & \\
\hline$\geq 2$ & $620(8.8)$ & $8(7)$ & $612(8.9)$ & \\
\hline Type & & & & $<0.001$ \\
\hline Budesonide & $1,674(23.8)$ & $19(16.7)$ & $1,655(23.9)$ & \\
\hline Fluticasone & $4,001(57.0)$ & $76(66.7)$ & $3,925(56.9)$ & \\
\hline Beclomethasone & $1,054(15.1)$ & $13(11.4)$ & $1,041(15.1)$ & \\
\hline Ciclesonide & $290(4.1)$ & $6(5.2)$ & $284(4.1)$ & \\
\hline
\end{tabular}

Values are presented as number (\%).

*MPR ratio was categorized into three adherence groups as follows: low adherence (MPR, $<50 \%)$, medium adherence (MPR, 50\%-79\%), and high adherence (MPR, $\geq 80 \%)$.

SARS-CoV-2: severe acute respiratory syndrome coronavirus 2; ICS: inhaled corticosteroid; MPR: medication possession rate.

Table 3. Multivariate analysis for inhaled corticosteroid use and risk of SARS-CoV-2

\begin{tabular}{|c|c|c|c|}
\hline Variable & Odds ratio* & 95\% Confidence interval & p-value \\
\hline \multicolumn{4}{|l|}{ ICS use } \\
\hline No & 1 & & \\
\hline Yes & 0.84 & $0.68-1.03$ & 0.089 \\
\hline \multicolumn{4}{|l|}{ ICS dose } \\
\hline No & 1 & & \\
\hline Low & 0.83 & $0.63-1.11$ & 0.204 \\
\hline Medium & 0.75 & $0.39-1.42$ & 0.373 \\
\hline High & 0.87 & $0.64-1.17$ & 0.347 \\
\hline \multicolumn{4}{|l|}{ ICS type } \\
\hline No & 1 & & \\
\hline Budesonide & 0.67 & $0.42-1.08$ & 0.098 \\
\hline Fluticasone & 0.86 & $0.67-1.09$ & 0.227 \\
\hline Beclomethasone & 0.88 & $0.50-1.56$ & 0.679 \\
\hline Ciclesonide & 1.33 & $0.58-3.09$ & 0.503 \\
\hline
\end{tabular}

*Adjusted for sex, age, region of residence, medical-aid beneficiary, respiratory disease, heart disease, diabetes mellitus, hypertension, and Charlson comorbidity index.

SARS-CoV-2: severe acute respiratory syndrome coronavirus 2; ICS: inhaled corticosteroid. 
ICS was not associated with positivity of SARS-CoV-2 infection in model 3.

\section{Comparison between ICS users and non-ICS users among those with positive SARS-CoV-2 infection}

Supplementary Table S2 shows comparison of clinical outcomes between ICS users and non-ICS users among those with positive SARS-CoV-2 infection. Medical cost, the proportion of hospital admission, the proportion of intensive care unit care, and all-cause mortality were not significantly different between the two groups.

\section{Discussion}

ICS plays an important role in controlling airway diseases such as asthma and COPD by reducing airway inflammation. However, the increased risk of infection associated with the use of ICS has always been a concern. Although the mechanism has not been fully elucidated, immunosuppressive effects of ICS are speculated to be associated with locally high concentration of ICS in the respiratory tract ${ }^{15}$. ICS is often regarded as being immunosuppressive. Its use is associated with an increased risk of respiratory infection. Thus, many physicians are concerned about an increased risk of SARS$\mathrm{CoV}-2$ infection in patients awith airway diseases taking ICS. Whether such patients should continue using ICS during the SARS-CoV-2 pandemic is unclear. Our study showed that ICS did not increase the positivity of SARS-CoV-2 infection. Furthermore, positivity of SARS-CoV-2 infection was not associated with the dose or the type of ICS.

Possible immunosuppressive effects of ICS have raised concerns about an increased risk of respiratory infection, including upper respiratory tract infection (URTI), pneumonia, and mycobacterial pulmonary disease. Two meta-analyses have reported that ICS use is associated with a significantly increased risk of URTI in patients with asthma or COPD ${ }^{16,17}$. Only high-dose ICS increased the risk of URTI (OR, 1.19) in patients with COPD, while low-dose (OR, 1.46) and high-dose (OR, 1.20) ICS increased the risk of URTI in patients with asth$\mathrm{ma}^{16,17}$. Singanayagam et al. ${ }^{18}$ have reported that ICS can impair innate and acquired antiviral immune responses and lead to delayed virus clearance. They also found previously unrecognized adverse effects such as enhanced mucus production, impaired antimicrobial peptide secretion, and increased pulmonary bacterial load during virus-induced exacerbations, which increased the risk of pneumonia after clearance of viral infection $^{18}$. Several studies and a meta-analysis have demonstrated an increased risk of pneumonia in COPD patients with ICS use, although this increased risk of pneumonia does not lead to a higher mortality ${ }^{6}$. However, ICS use and pneumonia in asthma patients have shown controversial results, which might be due to the varying age range and comorbidities assessed in different studies ${ }^{7,8,19}$. ICS can also increase the infection risk of both nontuberculous and tuberculous mycobacterial pulmonary diseases ${ }^{9,10,20}$. There is a strong dose-response relationship between mycobacterial infection and cumulative ICS dose ${ }^{20}$.

Recently, a similar study was conducted in 928 patients tested for SARS-CoV-2 at National Jewish Health ${ }^{21}$. There was no significant association between ICS use and testing positive for SARS-CoV-2 ${ }^{21}$. A meta-analysis has demonstrated that chronic use of ICS does not increase the risk for the development of a fatal course of SARS-CoV-2 ${ }^{22}$. In a large cohort of hospital admissions for SARS-CoV-2, patients aged 50 years and older with ICS use for asthma treatment showed lower mortality than those without underlying respiratory condition $^{23}$. However, patients with COPD had significantly increased mortality compared to those with no underlying respiratory condition regardless of ICS use ${ }^{23}$. Therefore, effects of ICS are expected to be different according to patient factors and types of chronic airway diseases.

Cell entry of coronaviruses depends on binding of viral spike (S) proteins to cellular receptors and S protein priming by host cell proteases. SARS-CoV-2 uses angiotensin converting enzyme 2 (ACE2) for entry and transmembrane protease serine 2 (TMPRSS2) for S protein priming ${ }^{24,25}$. An increased expression in lung cells will increase the susceptibility to SARS-CoV-2 infection or lead to a more severe COVID-19 disease $^{26}$.

Peters et al. ${ }^{26}$ have analyzed gene expression of ACE2 in sputum cells from 330 participants in the Severe Asthma Research Program-3 and 79 healthy controls. They found that the use of ICS was associated with decreased expression levels of ACE2 and TMPRSS2 $2^{26}$. However, intramuscular triamcinolone acetonide injection did not lower the expression level of either genes. This discordant result between systemic corticosteroid and inhaled corticosteroid might be related to assessment of sputum cell gene expression at different time points after exposure to corticosteroid ${ }^{26}$. They suggested that the decrease in ACE2 and TMPRSS2 gene expression provided some reassurance that ICS use would not increase the risk of SARS-CoV-2 infection or morbidity, although prospective studies are needed to confirm this assumption. Moreover, the mechanism by which ICS can reduce the expression of ACE2 and TMPRSS2 needs to be investigated further.

Types of ICS were not associated with positivity of SARSCoV-2. Iwabuchi et al. have reported cases of SARS-CoV-2 pneumonia successfully treated with ciclesonide inhalation ${ }^{27}$. However, it was unclear that whether these patients would have improved spontaneously because there was no control group in their study. Nevertheless, several reports have provided evidence for the beneficial effect of ICS on virus infections $^{28}$. Matsuyama et al. ${ }^{29}$ have shown that ICS ciclesonide can block coronavirus RNA replication by targeting 
viral NSP15. Moreover, glycopyrronium, formoterol, and a combination of glycopyrronium, formoterol, and budesonide can inhibit human coronaviruses-229E replication partly by inhibiting receptor expression and/or endosomal function, suggesting that these drugs may modulate infection-induced inflammation in the airway ${ }^{30}$.

Previous studies have reported that fluticasone use is associated with a higher risk of pneumonia than budesonide due to its greater and more protracted immunosuppressive effects locally in airways and lungs ${ }^{17,20}$. Local pharmacokinetic profiles (e.g., rate and extent of airway/pulmonary absorption) of ICS are strongly associated with intrinsic physicochemical properties of corticosteroids such as lipophilicity, aqueous solubility, and airway epithelial permeability ${ }^{31}$. However, ICS did not increase the positivity of SARS-CoV-2 infection in this study regardless the type of ICS. Differences in pharmacokinetics and immune modulatory effects of different types of ICS warrant further studies to investigate the relationship between different types of ICS and the positivity of SARS-CoV-2 infection.

This report provides further evidence on the relationship between the use of ICS and positivity of SARS-CoV-2 infection in a large population-based study including different types of ICS. However, this study has several limitations. The severity of primary respiratory diseases for which ICS were prescribed was not categorized. The role of ICS is different between asthma and COPD. ICS is an essential maintenance treatment in asthma while it is an add-on treatment in COPD with frequent exacerbations. Therefore, not just ICS, but also the severity of chronic respiratory disease might have affected the degree of positivity for SARS-CoV-2 infection. Secondly, previous use of systemic corticosteroid was not included in the analysis. Systemic corticosteroids are more potent immune modulators than ICS. Therefore, their effects on positivity for SARS-CoV-2 infection are also important. Thirdly, the positivity of infection based on different types of ICS could not be fully evaluated because different numbers of each ICS were prescribed. Lastly, this study included people who were suspected of or at a high risk of SARS-CoV-2 infection. Therefore, asymptomatic infected persons might have been missed. Moreover, data analyzed were from the administrative claims database until the middle of the pandemic period. Thus, this study could not represent the whole population who were suspected of having SARS-CoV-2, at a high risk of infection, and confirmed with SARS-CoV-2 infection in Korea.

In conclusion, prior use of ICS within one year did not increase the positivity for SARS-CoV-2 infection in Korea. Furthermore, different doses or types of ICS did not affect the the positivity of SARS-CoV-2 infection. The current guidelines recommend no changes to the treatment or management of chronic respiratory conditions including asthma and COPD and suggest continuing the use of ICS even during the COVID-19 pandemic. Further epidemiologic studies with a large population are warranted to clarify the association between ICS use and the risk of SARS-CoV-2 infection as well as its mechanisms.

\section{Authors' Contributions}

Conceptualization: Lee SC, Park SC, Jung JY. Methodology: Lee SC. Data curation: Son KJ. Writing - original draft preparation: Park SC, Jung JY. Writing - review and editing: Han CS, Park SC, Jung JY. Approval of final manuscript: all authors.

\section{Conflicts of Interest}

No potential conflict of interest relevant to this article was reported.

\section{Acknowledgments}

The authors appreciate the contribution of healthcare professionals dedicated to treating SARS-CoV-2 patients in Korea and the Ministry of Health and Welfare and the Health Insurance Review \& Assessment Service of Korea for sharing invaluable national health insurance claims data in a prompt manner.

\section{Funding}

This work was supported by a grant (No. 2019R1F1Al 061841) of the National Research Foundation of Korea (NRF) funded by the Korea government (Ministry of Science and ICT).

\section{Supplementary Material}

Supplementary material can be found in the journal homepage (http://www.e-trd.org).

Supplementary Table S1. Multivariate analysis for positivity of SARS-CoV-2.

Supplementary Table S2. Comparison between ICU users and non-ICS users among those with positive SARS-CoV-2 infection.

\section{References}

1. Zhu N, Zhang D, Wang W, Li X, Yang B, Song J, et al. A Novel Coronavirus from Patients with Pneumonia in China, 2019. N Engl J Med 2020;382:727-33. 
2. World Health Organization. Coronavirus disease (COVID-2019) situation reports. Geneva: World Health Organization; 2020.

3. Chen N, Zhou M, Dong X, Qu J, Gong F, Han Y, et al. Epidemiological and clinical characteristics of 99 cases of 2019 novel coronavirus pneumonia in Wuhan, China: a descriptive study. Lancet 2020;395:507-13.

4. Guan WJ, Ni ZY, Hu Y, Liang WH, Ou CQ, He JX, et al. Clinical characteristics of coronavirus disease 2019 in China. N Engl J Med 2020;382:1708-20.

5. Yang J, Zheng Y, Gou X, Pu K, Chen Z, Guo Q, et al. Prevalence of comorbidities and its effects in patients infected with SARS-CoV-2: a systematic review and meta-analysis. Int J Infect Dis 2020;94:91-5.

6. Kew KM, Seniukovich A. Inhaled steroids and risk of pneumonia for chronic obstructive pulmonary disease. Cochrane Database Syst Rev 2014;(3):CD010115.

7. McKeever T, Harrison TW, Hubbard R, Shaw D. Inhaled corticosteroids and the risk of pneumonia in people with asthma: a case-control study. Chest 2013;144:1788-94.

8. Kim MH, Rhee CK, Shim JS, Park SY, Yoo KH, Kim BY, et al. Inhaled corticosteroids in asthma and the risk of pneumonia. Allergy Asthma Immunol Res 2019;11:795-805.

9. Lee CH, Kim K, Hyun MK, Jang EJ, Lee NR, Yim JJ. Use of inhaled corticosteroids and the risk of tuberculosis. Thorax 2013;68:1105-13.

10. Andrejak C, Nielsen R, Thomsen VO, Duhaut P, Sorensen HT, Thomsen RW. Chronic respiratory disease, inhaled corticosteroids and risk of non-tuberculous mycobacteriosis. Thorax 2013;68:256-62.

11. Lam WY, Fresco P. Medication adherence measures: an overview. Biomed Res Int 2015;2015:217047.

12. Engelkes M, Janssens HM, de Jongste JC, Sturkenboom MC, Verhamme KM. Medication adherence and the risk of severe asthma exacerbations: a systematic review. Eur Respir J 2015;45:396-407.

13. Charlson ME, Pompei P, Ales KL, MacKenzie CR. A new method of classifying prognostic comorbidity in longitudinal studies: development and validation. J Chronic Dis 1987;40:373-83.

14. Global Initiative for Asthma. Global strategy for asthma management and prevention. Fontana: Global Initiative for Asthma; 2020.

15. Suissa S, McGhan R, Niewoehner D, Make B. Inhaled corticosteroids in chronic obstructive pulmonary disease. Proc Am Thorac Soc 2007;4:535-42.

16. Yang M, Chen H, Zhang Y, Du Y, Xu Y, Jiang P, et al. Long-term use of inhaled corticosteroids and risk of upper respiratory tract infection in chronic obstructive pulmonary disease: a meta-analysis. Inhal Toxicol 2017;29:219-26.

17. Yang M, Zhang Y, Chen H, Lin J, Zeng J, Xu Z. Inhaled corticosteroids and risk of upper respiratory tract infection in patients with asthma: a meta-analysis. Infection 2019;47:377-85.
18. Singanayagam A, Glanville N, Girkin JL, Ching YM, Marcellini A, Porter JD, et al. Corticosteroid suppression of antiviral immunity increases bacterial loads and mucus production in COPD exacerbations. Nat Commun 2018;9:2229.

19. O’Byrne PM, Pedersen S, Carlsson LG, Radner F, Thoren A, Peterson S, et al. Risks of pneumonia in patients with asthma taking inhaled corticosteroids. Am J Respir Crit Care Med 2011;183:589-95.

20. Brode SK, Campitelli MA, Kwong JC, Lu H, Marchand-Austin A, Gershon AS, et al. The risk of mycobacterial infections associated with inhaled corticosteroid use. Eur Respir J 2017;50: 1700037.

21. Liao SY, Petrache I, Fingerlin TE, Maier LA. Association of inhaled and systemic corticosteroid use with Coronavirus Disease 2019 (COVID-19) test positivity in patients with chronic pulmonary diseases. Respir Med 2021;176:106275.

22. Kow CS, Hasan SS. Preadmission use of inhaled corticosteroids and risk of fatal or severe COVID-19: a meta-analysis. J Asthma 2021 Feb 8 [Epub]. https://doi.org/10.1080/02770903. 2021.1878531.

23. Bloom CI, Drake TM, Docherty AB, Lipworth BJ, Johnston SL, Nguyen-Van-Tam JS, et al. Risk of adverse outcomes in patients with underlying respiratory conditions admitted to hospital with COVID-19: a national, multicentre prospective cohort study using the ISARIC WHO Clinical Characterisation Protocol UK. Lancet Respir Med 2021;9:699-711.

24. Yan R, Zhang Y, Li Y, Xia L, Guo Y, Zhou Q. Structural basis for the recognition of SARS-CoV-2 by full-length human ACE2. Science 2020;367:1444-8.

25. Hoffmann M, Kleine-Weber H, Schroeder S, Kruger N, Herrler T, Erichsen S, et al. SARS-CoV-2 cell entry depends on ACE2 and TMPRSS 2 and is blocked by a clinically proven protease inhibitor. Cell 2020;181:271-80.

26. Peters MC, Sajuthi S, Deford P, Christenson S, Rios CL, Montgomery MT, et al. COVID-19-related genes in sputum cells in asthma: relationship to demographic features and corticosteroids. Am J Respir Crit Care Med 2020;202:83-90.

27. Iwabuchi K, Yoshie K, Kurakami Y, Takahashi K, Kato Y, Morishima T. Therapeutic potential of ciclesonide inahalation for COVID-19 pneumonia: report of three cases. J Infect Chemother 2020;26:625-32.

28. Halpin DM, Faner R, Sibila O, Badia JR, Agusti A. Do chronic respiratory diseases or their treatment affect the risk of SARSCoV-2 infection? Lancet Respir Med 2020;8:436-8.

29. Matsuyama S, Kawase M, Nao N, Shirato K, Ujike M, Kamitani $\mathrm{W}$, et al. The inhaled corticosteroid ciclesonide blocks coronavirus RNA replication by targeting viral NSP15. Preprint at https://doi.org/10.1101/2020.03.11.987016 (2020).

30. Yamaya M, Nishimura H, Deng X, Sugawara M, Watanabe O, Nomura K, et al. Inhibitory effects of glycopyrronium, formoterol, and budesonide on coronavirus $\mathrm{HCoV}-229 \mathrm{E}$ replication and cytokine production by primary cultures of human nasal and tracheal epithelial cells. Respir Investig 
2020;58:155-68.

31. Janson C, Stratelis G, Miller-Larsson A, Harrison TW, Larsson K. Scientific rationale for the possible inhaled corticosteroid intraclass difference in the risk of pneumonia in COPD. Int J Chron Obstruct Pulmon Dis 2017;12:3055-64. 